\title{
Incidence of and risk factors for preoperative deep venous thrombosis in patients undergoing gastric cancer surgery
}

\author{
Takeyuki Wada $^{1} \cdot$ Hisataka Fujiwara ${ }^{1} \cdot$ Shinji Morita $^{1} \cdot$ Takeo Fukagawa $^{1} \cdot$ \\ Hitoshi Katai ${ }^{1}$
}

Received: 30 July 2016/ Accepted: 9 January 2017/Published online: 24 January 2017

(c) The International Gastric Cancer Association and The Japanese Gastric Cancer Association 2017

\begin{abstract}
Background Pulmonary thromboembolism (PE) is one of the life-threatening complications of gastric cancer surgery. D-dimer assay is a safe and rapid tool to exclude the presence of deep venous thrombosis (DVT). In July 2012, we started preoperative DVT screening of patients scheduled for gastric cancer surgery using a combination of D-dimer measurements and lower extremity venous ultrasonography to prevent PE.

Methods Between July 2012 and August 2015, 976 consecutive patients underwent gastric cancer surgery with preoperative D-dimer screening. Lower extremity venous ultrasonography was performed in patients with a positive D-dimer assay result (greater than $1.0 \mu \mathrm{g} / \mathrm{ml}$ ). The incidence of and risk factors for preoperative DVT and the incidence of PE were examined in patients undergoing gastric cancer surgery.

Results Of the 976 patients, 176 (18.0\%) showed positive D-dimer assay results, and in $13(1.3 \%)$ DVT was diagnosed by lower extremity ultrasonography. Our analysis identified neoadjuvant chemotherapy as a risk factor for preoperative detection of DVT in patients undergoing gastric cancer surgery $(P=0.021)$. The incidence of PE was $0.1 \%$ (1/976). Conclusion Preoperative gastric cancer patients receiving neoadjuvant chemotherapy seem to be at higher risk for the development of DVT.
\end{abstract}

Keywords Gastric cancer - Deep venous thrombosis · D-dimer

Hitoshi Katai

hkatai@ncc.go.jp

1 Gastric Surgery Division, National Cancer Center Hospital, 5-1-1 Tsukiji, Chuo-Ku, Tokyo 104-0045, Japan

\section{Introduction}

Gastric cancer is the third commonest cause of cancer-related death in the world [1]. Surgery is the main curative treatment for resectable gastric cancer. Pulmonary thromboembolism (PE) is one of the life-threatening complications of gastric cancer surgery. Because both the cancer per se and surgery are known to be independent risk factors for the development of venous thromboembolism (VTE), routine thromboprophylaxis is recommended in patients undergoing cancer surgery [2]. Intermittent pneumatic compression (IPC) is usually used during and after surgery to prevent the complication of PE. However, in patients with preexisting deep venous thrombosis (DVT), IPC may be ineffective and possibly lead to fatal PE. The D-dimer assay is a safe and useful tool with a high sensitivity (97-100\%) for excluding DVT and a high negative predictive value (96-100\%) [3-5]. Lower extremity venous ultrasonography is the standard method for the diagnosis of DVT, with a high sensitivity (93-96\%) and high specificity (98-99\%) [6-8]. In July 2012, we started preoperative DVT screening at our institution using a combination of D-dimer measurements and lower extremity venous ultrasonography in patients undergoing gastric cancer surgery. In the present study, we investigated the incidence of and risk factors for DVT and the incidence of $\mathrm{PE}$ in patients undergoing gastric cancer surgery.

\section{Patients and methods}

\section{Patients}

We started conducting serum D-dimer measurements for all gastric cancer patients scheduled for surgery in July 2012. Nine hundred seventy-six consecutive gastric cancer 
Fig. 1 Flowchart for routine thromboprophylaxis. $D D$ D-dimer, $D V T$ deep venous thrombosis

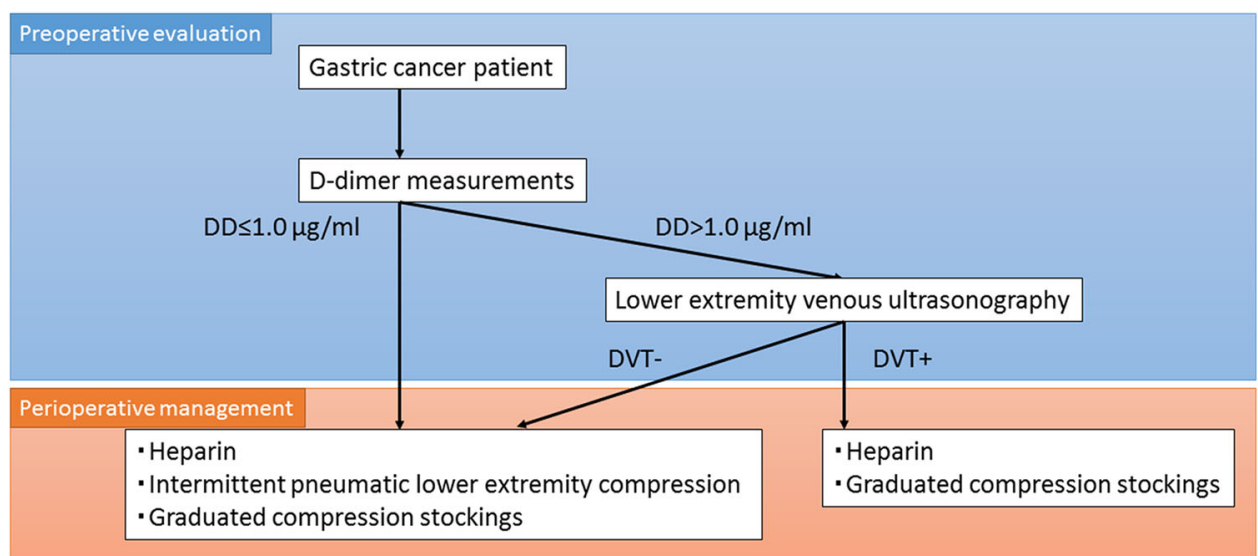

patients who underwent preoperative D-dimer screening between July 2012 and August 2015 at our hospital were included in this study. The incidence of and risk factors for DVT and the incidence of PE were investigated in the patients undergoing surgery for gastric cancer.

The TNM stages were recorded according to the Japanese classification of gastric carcinoma [9]. Papillary adenocarcinoma and well-differentiated or moderately differentiated tubular adenocarcinoma were categorized as differentiated-type carcinoma, whereas poorly differentiated adenocarcinoma, signet ring cell carcinoma, and mucinous adenocarcinoma were categorized as undifferentiated-type carcinoma. Patients with other pathological types of gastric cancer were excluded from the study.

All patients underwent gastrectomy with curative intent. The gastric resection was classified as total, distal, proximal or pylorus-preserving gastrectomy. Patients undergoing completion gastrectomy for remnant gastric cancer were also included in the study, whereas those undergoing wedge resection were excluded. The extent of lymphadenectomy performed depended on the type of resection and the stage of the gastric cancer classified in accordance with the Japanese classification [9]. Those who needed simultaneous surgery for other synchronous cancer were excluded.

Neoadjuvant therapy with a combination of S-1 plus cisplatin was initiated for patients with apparent para-aortic node metastasis or bulky nodal metastases surrounding the celiac artery or its branches.

Preoperative medical comorbidities were investigated especially for renal disease, pulmonary disease, and arterial thromboembolism. These comorbidities have been recognized as risk factors for cancer-associated thrombosis in the guideline in [10]. The International Classification of Diseases, Ninth Revision, Clinical Modification (ICD-9-CM) was used to diagnose the comorbidity [renal disease (ICD9-CM codes 580-593), pulmonary disease (ICD-9-CM codes 487-519), arterial thromboembolism (ICD-9-CM code 444)] with similar uses as the guideline in [10].

\section{Thromboprophylaxis}

D-dimer assay was performed in all gastric cancer patients scheduled to undergo gastric cancer surgery. Lower extremity venous ultrasonography was performed in those patients who had a positive the D-dimer assay result (greater than $1.0 \mu \mathrm{g} / \mathrm{ml}$ ).

Routine thromboprophylaxis (heparin, IPC, and compression stockings) was used for patients who had a negative result of preoperative screening for DVT. IPC was withheld during and after surgery in patients in whom DVT was detected during the preoperative screening. All patients received preoperative fluid infusion $(80 \mathrm{ml} / \mathrm{h})$ from the evening before surgery. Low-dose unfractionated heparin was administered within $2 \mathrm{~h}$ after surgery as a continuous intravenous infusion at the rate of $10,000 \mathrm{IU} / \mathrm{day}$ until the second postoperative day. This anticoagulation was omitted for patients with coagulopathy or a high risk of postoperative bleeding. IPC was performed intraoperatively and postoperatively until the patient resumed walking, which was usually on the first postoperative day. Graduated compression stockings were also used from the morning of surgery until the patient started walking adequately. Thus, all thromboprophylaxis modalities were discontinued by the second postoperative day, unless the patient was still unable to walk by then for some reason. A thromboprophylaxis flowchart is shown in Fig. 1. Inferior vena cava filter was temporarily used during the perioperative period for patients with PE incidentally discovered by preoperative computed tomography scan if they had a contraindication to anticoagulation or if they had PE despite adequate anticoagulation.

\section{Diagnosis of PE}

A prospective database was used to identify patients who developed the complication of PE postoperatively during hospitalization. Clinically suspected PE was confirmed by 
contrast-enhanced helical computed tomography. Only those cases of PE that required some medical intervention (Common Terminology Criteria for Adverse Events grade 3 or higher [11]) were counted. Patients with incidentally detected asymptomatic postoperative PE were excluded from this study.

\section{Statistical analysis}

Continuous variables are presented as the median and range. Proportions were compared with the $\chi^{2}$ test or Fisher's exact test (when the frequencies were less than 5), and continuous variables were compared with Student's $t$ test. Analyses were performed with IBM SPSS Statistics, version 22. $P<0.05$ was considered to represent statistical significance.

\section{Results}

\section{Patient characteristics}

The enrolled patients' characteristics are listed in Table 1. The median age of the patients was 67 years (range 27-90 years) and the median body mass index was $23 \mathrm{~kg} /$ $\mathrm{m}^{2}$ (range $15-38 \mathrm{~kg} / \mathrm{m}^{2}$ ). There were 628 patients $(64 \%)$ with stage I cancer, 165 patients (17\%) with stage II cancer, 153 patients (16\%) with stage IIII cancer, and 30 patients (3\%) with stage IV cancer. Forty-seven patients (5\%) received neoadjuvant chemotherapy. Renal disease, pulmonary disease, and arterial thromboembolism were seen in 14 patients (1\%), 34 patients (3\%), and 33 patients (3\%) respectively. Incidentally detected preoperative PE was seen in two patients. Inferior vena cava filter was used for one patient, because his PE was detected despite adequate anticoagulation for another disease. Another patient with preoperative $\mathrm{PE}$ received only anticoagulation therapy.

\section{D-dimer test positive rate and incidence of DVT and PE}

Of the 976 patients registered, $176(18.0 \%)$ had a positive D-dimer assay result. DVT was detected in 13 patients (1.3\% of all patients, $7.4 \%$ of D-dimer-positive patients). One D-dimer-positive patient $(0.1 \%)$ who did not have DVT developed PE in this series, and she recovered with systemic anticoagulant therapy. Exacerbation of PE was not seen in the two patients with incidentally discovered preoperative PE. The preoperative D-dimer assay result was positive in those two patients.
Table 1 Patient characteristics $(n=976)$

\begin{tabular}{|c|c|}
\hline Parameter & Value \\
\hline \multicolumn{2}{|l|}{ Age (years) } \\
\hline Median & 67 \\
\hline Range & $27-90$ \\
\hline \multicolumn{2}{|l|}{ Sex } \\
\hline Male & $631(65 \%)$ \\
\hline Female & $345(35 \%)$ \\
\hline \multicolumn{2}{|l|}{ BMI $\left(\mathrm{kg} / \mathrm{m}^{2}\right)$} \\
\hline Median & 23 \\
\hline Range & $15-38$ \\
\hline \multicolumn{2}{|l|}{ Histological type } \\
\hline Differentiated & $440(45 \%)$ \\
\hline Undifferentiated & $536(55 \%)$ \\
\hline \multicolumn{2}{|l|}{ Neoadjuvant chemotherapy } \\
\hline Yes & $47(5 \%)$ \\
\hline No & $929(95 \%)$ \\
\hline \multicolumn{2}{|l|}{ Comorbidities } \\
\hline Renal disease & $14(1 \%)$ \\
\hline Pulmonary disease & $34(3 \%)$ \\
\hline Arterial thromboembolism & $33(3 \%)$ \\
\hline Incidentally discovered preoperative PE & $2(0.2 \%)$ \\
\hline \multicolumn{2}{|l|}{ Type of surgery } \\
\hline Total gastrectomy & $227(23 \%)$ \\
\hline Distal gastrectomy & $448(46 \%)$ \\
\hline Proximal gastrectomy & $65(7 \%)$ \\
\hline Pylorus-preserving gastrectomy & $208(21 \%)$ \\
\hline Completion gastrectomy & $28(3 \%)$ \\
\hline \multicolumn{2}{|l|}{ Open or laparoscopic surgery } \\
\hline Open & $833(85 \%)$ \\
\hline Laparoscopic & $143(15 \%)$ \\
\hline \multicolumn{2}{|l|}{ Blood loss (ml) } \\
\hline Median & 155 \\
\hline Range & $1-5275$ \\
\hline \multicolumn{2}{|l|}{ Operation time (min) } \\
\hline Median & 258 \\
\hline Range & $120-727$ \\
\hline \multicolumn{2}{|l|}{ Pathological stage } \\
\hline I & $628(64 \%)$ \\
\hline II & $165(17 \%)$ \\
\hline III & $153(16 \%)$ \\
\hline IV & $30(3 \%)$ \\
\hline
\end{tabular}

$P E$ pulmonary thromboembolism

\section{Risk factors for DVT}

Univariate analysis was performed with the variables of age, sex (male/female), body mass index, histopathological type, $\mathrm{T}$ category ( $\mathrm{T} 1$ or $\mathrm{T} 2$ or greater), $\mathrm{N}$ category ( $\mathrm{N} 0$ or 
Table 2 Risk factors for deep venous thrombosis $(D V T)$

\begin{tabular}{|c|c|c|c|}
\hline Factor & $\begin{array}{l}\text { DVT } \\
\text { positive }(n=13)\end{array}$ & $\begin{array}{l}\text { DVT } \\
\text { negative }(n=963)\end{array}$ & $P$ \\
\hline Age & 77 & 67 & 0.159 \\
\hline Sex (male/female) & $8 / 5$ & $623 / 340$ & 0.511 \\
\hline BMI & 24 & 23 & 0.691 \\
\hline $\begin{array}{l}\text { Histopathological type (differentiated/ } \\
\text { undifferentiated) }\end{array}$ & $3 / 10$ & $437 / 526$ & 0.108 \\
\hline $\mathrm{pT}(1 / \geq 2)$ & $5 / 8$ & $572 / 391$ & 0.127 \\
\hline $\mathrm{pN}(0 / \geq 1)$ & $9 / 4$ & $669 / 294$ & 0.597 \\
\hline Pathological stage (I/ $\geq$ II) & $6 / 7$ & $621 / 342$ & 0.242 \\
\hline Neoadjuvant chemotherapy (yes/no) & $3 / 10$ & $43 / 920$ & 0.021 \\
\hline Renal disease (yes/no) & $0 / 13$ & $14 / 949$ & 0.828 \\
\hline Pulmonary disease (yes/no) & $1 / 12$ & $33 / 930$ & 0.371 \\
\hline Arterial thromboembolism (yes/no) & $1 / 12$ & $32 / 931$ & 0.362 \\
\hline
\end{tabular}

N1 or greater), pathological stage (I or II or greater), neoadjuvant chemotherapy, and comorbidities. The analysis identified neoadjuvant chemotherapy $(P=0.021)$ as a significant risk factor for the detection of preoperative DVT in patients scheduled for gastric cancer surgery (Table 2).

\section{Discussion}

$\mathrm{PE}$ is one of the potentially fatal complications of gastric cancer surgery. DVT often develops in the deep veins of the leg and potentially causes PE when clots break off from the thrombi on the vein walls and travel through the heart to the pulmonary arteries. The epidemiological and clinical relevance of the association between VTE and cancer is well documented. Patients with cancer have an increased risk of development of VTE as compared with the general population [12, 13]. The need for appropriate prophylaxis against PE in patients scheduled for cancer surgery has been increasingly recognized. Guidelines recommend both pharmacological and mechanical modalities for perioperative thromboprophylaxis in patients undergoing major cancer surgery [10]. At our hospital, we have adopted lowdose unfractionated heparin and IPC as the pharmacological and mechanical modalities, respectively, of perioperative thromboprophylaxis. However, in cases where DVT exists before surgery, IPC may be ineffective and may even increase the risk of fatal PE. Although the guideline recommends 7-10 days of pharmacologic thromboprophylaxis for patients undergoing major surgery for cancer, it is also stated that the appropriate duration of thromboprophylaxis in lower-risk patients should be decided on a caseby-case basis [10]. Thromboprophylaxis in our hospital is discontinued by the second postoperative day, because Asians are considered to be lower-risk patients and we have already reported low PE incidence with this thromboprophylaxis [14].

Although the D-dimer assay has a high negative predictive value (96-100\%), which allows exclusion of VTE [3-5], its specificity is only 36-44\% [15, 16]. On the other hand, lower extremity venous ultrasonography has high sensitivity (93-96\%) as well as high specificity (98-99\%) for the diagnosis of DVT [6-8]. However, ultrasonography in all preoperative patients as a screening tool is considered to represent overuse of this diagnostic resource [17, 18]. Appropriate use of the D-dimer assay limits the overuse and added cost of ultrasonography without a negative impact [17]. Therefore, we use the D-dimer assay in combination with ultrasonography at our hospital to screen patients for the existence of DVT preoperatively.

Our preoperative DVT screening of gastric cancer patients using the D-dimer assay in combination with ultrasonography revealed a DVT incidence of $1.3 \%$. This value may not be entirely accurate, because ultrasonography was not performed in all patients. Therefore, this study is an incomplete cohort study to determine the real incidence of DVT in gastric cancer surgery. It remains unclear whether there was no case of DVT in D-dimer-negative patients. However, its reliability can be considered to be acceptable, taking into account the high sensitivity and high negative predictive value of the D-dimer assay and the high sensitivity and high specificity of ultrasonography, which have already been reported [3-8]. Moreover, even in D-dimer-positive cases, only $7.4 \%$ of patients had DVT. Ultrasonography for all patients before surgery is not justified in Japan. This combined screening method seems to be effective to detect DVT in gastric cancer patients scheduled for surgery. Larsen et al. [19] investigated the incidence of DVT in esophageal and gastric cancer patients, and reported that the incidence of DVT in gastric cancer patients was $37 \%$ (10 of 27 patients). A higher 
proportion of patients with advanced cancer in their study and the racial differences between their study and ours presumably explains the discrepancy between their results and ours. Kawaguchi et al. [20] reported that the cutoff value of the serum D-dimer level to diagnose DVT before ovarian cancer treatment was $1.5 \mu \mathrm{g} / \mathrm{ml}$. The corresponding cutoff value in the gastric cancer patients scheduled for surgery could not be identified in this study, because ultrasonography was not performed in all patients; therefore, evaluation of this cutoff value remains for a future study.

Neoadjuvant chemotherapy was identified as a risk factor for the preoperative detection of DVT in gastric cancer patients scheduled for surgery in the present study. This result is consistent with the report that chemotherapy and hospitalization are independent risk factors for VTE in cancer patients [21]. Although a randomized controlled trial is required to deny the influence of some bias factors such as para-aortic node metastasis or bulky nodal metastases, it is considered impossible to carry out a randomized controlled trial because the importance of neoadjuvant chemotherapy for advanced gastric cancer with extensive lymph node metastasis was reported [22] and it has already been recognized as the standard treatment. In view of the recent report of the importance of neoadjuvant chemotherapy in patients with advanced gastric cancer [23-25], particular attention to preoperative detection of DVT is required in gastric cancer patients scheduled for surgical treatment. Medical comorbidities were not identified as risk factor in this study, probably because this study was planned for perioperative patients and consisted mainly of patients without severe comorbidities.

PE occurred in only one patient in this study. She had received neoadjuvant chemotherapy. A patient who has received neoadjuvant chemotherapy, which was suggested as the risk factor for DVT in this study, should be carefully treated considering the possibility of thrombotic disease. We previously reported an incidence of $\mathrm{PE}$ in gastric cancer surgery patients of $0.2 \%$ (7 of 3262 patients) [14]. DVT screening was not performed in that study. The lower incidence of PE in the present study was possibly achieved because of the screening or patients for DVT, although this would not be a statistically proper comparison. The main purpose of this study was to investigate the incidence of and risk factors for DVT in gastric cancer surgery. However, it also seems important that the lower incidence of PE was achieved by this combination screening program of D-dimer measurements and lower extremity venous ultrasonography.

In conclusion, gastric cancer patients scheduled for surgery receiving neoadjuvant chemotherapy seem to be at a higher risk of DVT.

\section{Compliance with ethical standards}

Conflict of interest The authors declare that they have no conflict of interest.

Human rights statement and informed consent All procedures followed were in accordance with the ethical standards of the responsible committee on human experimentation (institutional and national) and with the Helsinki Declaration of 1964 and later versions. Informed consent or substitute for it was obtained from all patients for their being included in the study.

\section{References}

1. Ferlay J, Soerjomataram I, Dikshit R, Eser S, Mathers C, Rebelo $\mathrm{M}$, et al. Cancer incidence and mortality worldwide: sources, methods and major patterns in GLOBOCAN 2012. Int J Cancer. 2015;136(5):E359-86. doi:10.1002/ijc.29210.

2. Geerts WH, Bergqvist D, Pineo GF, Heit JA, Samama CM, Lassen MR, et al. Prevention of venous thromboembolism: american college of chest physicians evidence-based clinical practice guidelines. Chest. 2008;133:381S-453S. doi:10.1378/ chest.08-0656.

3. Funfsinn N, Caliezi C, Biasiutti FD, Korte W, Z'Brun A, Baumgartner I, et al. Rapid D-dimer testing and pre-test clinical probability in the exclusion of deep venous thrombosis in symptomatic outpatients. Blood Coagul Fibrinolysis. 2001;12(3):165-70.

4. Legnani C, Pancani C, Palareti G, Guazzaloca G, Coccheri S. Contribution of a new, rapid, quantitative and automated method for D-dimer measurement to exclude deep vein thrombosis in symptomatic outpatients. Blood Coagul Fibrinolysis. 1999;10(2):69-74.

5. van der Graaf F, van den Borne H, van der Kolk M, de Wild PJ, Janssen GW, van Uum SH. Exclusion of deep venous thrombosis with D-dimer testing-comparison of 13 D-dimer methods in 99 outpatients suspected of deep venous thrombosis using venography as reference standard. Thromb Haemost. 2000;83(2):191-8.

6. White RH, McGahan JP, Daschbach MM, Hartling RP. Diagnosis of deep-vein thrombosis using duplex ultrasound. Ann Intern Med. 1989;111(4):297-304.

7. Becker DM, Philbrick JT, Abbitt PL. Real-time ultrasonography for the diagnosis of lower extremity deep venous thrombosis. The wave of the future? Arch Intern Med. 1989;149(8):1731-4.

8. Cogo A, Lensing AW, Wells P, Prandoni P, Buller HR. Noninvasive objective tests for the diagnosis of clinically suspected deep-vein thrombosis. Haemostasis. 1995;25(1-2):27-39.

9. Japanese Gastric Cancer Association. Japanese classification of gastric carcinoma: 3rd English edition. Gastric Cancer. 2011;14:101-12. doi:10.1007/s10120-011-0041-5.

10. Lyman GH, Khorana AA, Kuderer NM, Lee AY, Arcelus JI, Balaban EP, et al. Venous thromboembolism prophylaxis and treatment in patients with cancer: American Society of Clinical Oncology clinical practice guideline update. J Clin Oncol. 2013;31(17):2189-204. doi:10.1200/JCO.2013.49.1118.

11. National Cancer Institute. Common Terminology Criteria for Adverse Events (CTCAE) v4.0. 2010. https://evs.nci.nih.gov/ ftp1/CTCAE/CTCAE_4.03_2010-06-14_QuickReference_5x7. pdf.

12. Blom JW, Doggen CJ, Osanto S, Rosendaal FR. Malignancies, prothrombotic mutations, and the risk of venous thrombosis. JAMA. 2005;293(6):715-22. doi:10.1001/jama.293.6.715.

13. Heit JA, Silverstein MD, Mohr DN, Petterson TM, O'Fallon WM, Melton LJ 3rd. Risk factors for deep vein thrombosis and 
pulmonary embolism: a population-based case-control study. Arch Intern Med. 2000;160(6):809-15.

14. Saka M, Morita S, Fukagawa T, Nijjar R, Katai H. Incidence of pulmonary thromboembolism in gastric cancer surgery using routine thromboprophylaxis. Gastric Cancer. 2010;13(2):117-22. doi:10.1007/s10120-010-0551-6.

15. Larsen TB, Stoffersen E, Christensen CS, Laursen B. Validity of D-dimer tests in the diagnosis of deep vein thrombosis: a prospective comparative study of three quantitative assays. J Intern Med. 2002;252(1):36-40.

16. Michiels JJ, Maasland H, Moossdorff W, Lao M, Gadiseur A, Schroyens W. Safe exclusion of deep vein thrombosis by a rapid sensitive ELISA D-dimer and compression ultrasonography in 1330 outpatients with suspected DVT. Angiology. 2016;67(8):781-7. doi:10.1177/0003319715616007.

17. Mousa AY, Broce M, Gill G, Kali M, Yacoub M, AbuRahma AF. Appropriate use of D-dimer testing can minimize over-utilization of venous duplex ultrasound in a contemporary high-volume hospital. Ann Vasc Surg. 2015;29(2):311-7. doi:10.1016/j.avsg. 2014.07.032..

18. Wells PS, Anderson DR, Rodger M, Forgie M, Kearon C, Dreyer $\mathrm{J}$, et al. Evaluation of D-dimer in the diagnosis of suspected deepvein thrombosis. N Engl J Med. 2003;349(13):1227-35. doi:10. 1056/NEJMoa023153.

19. Larsen AC, Frokjaer JB, Fisker RV, Iyer V, Mortensen PB, Yilmaz MK, et al. Treatment-related frequency of venous thrombosis in lower esophageal, gastro-esophageal and gastric cancer-a clinical prospective study of outcome and prognostic factors. Thromb Res. 2015;135(5):802-8. doi:10.1016/j.thromres. 2015.01.021.

20. Kawaguchi R, Furukawa N, Kobayashi H. Cut-off value of D-dimer for prediction of deep venous thrombosis before treatment in ovarian cancer. J.Gynecol Oncol. 2012;23(2):98-102. doi:10.3802/jgo.2012.23.2.98.

21. Ashrani AA, Gullerud RE, Petterson TM, Marks RS, Bailey KR, Heit JA. Risk factors for incident venous thromboembolism in active cancer patients: a population based case-control study. Thromb Res. 2016;139:29-37. doi:10.1016/j.thromres.2016.01. 002.

22. Yoshikawa T, Sasako M, Yamamoto S, Sano T, Imamura H, Fujitani K, et al. Phase II study of neoadjuvant chemotherapy and extended surgery for locally advanced gastric cancer. Br J Surg. 2009;96(9):1015-22. doi:10.1002/bjs.6665.

23. Tsuburaya A, Mizusawa J, Tanaka Y, Fukushima N, Nashimoto A, Sasako M, et al. Neoadjuvant chemotherapy with S-1 and cisplatin followed by D2 gastrectomy with para-aortic lymph node dissection for gastric cancer with extensive lymph node metastasis. Br J Surg. 2014;101(6):653-60. doi:10.1002/bjs.9484.

24. Yoshikawa T, Morita S, Tanabe K, Nishikawa K, Ito Y, Matsui T, et al. Survival results of a randomised two-by-two factorial phase II trial comparing neoadjuvant chemotherapy with two and four courses of S-1 plus cisplatin (SC) and paclitaxel plus cisplatin (PC) followed by D2 gastrectomy for resectable advanced gastric cancer. Eur J Cancer. 2016;62:103-11. doi:10.1016/j.ejca.2016. 04.012.

25. Inoue K, Nakane Y, Kogire M, Fujitani K, Kimura Y, Imamura $\mathrm{H}$, et al. Phase II trial of preoperative S-1 plus cisplatin followed by surgery for initially unresectable locally advanced gastric cancer. Eur J Surg Oncol. 2012;38(2):143-9. doi:10.1016/j.ejso. 2011.11.009. 